\title{
p.D1690N sodium voltage-gated channel $\alpha$ subunit 5 mutation reduced sodium current density and is associated with Brugada syndrome
}

\author{
ZHIPENG ZENG ${ }^{1,2^{*}}$, QIANG XIE ${ }^{2 *}$, YUAN HUANG $^{3}$, YUANYUAN ZHAO ${ }^{3}$, \\ WEIHUA $\mathrm{LI}^{2}$ and ZHENGRONG HUANG ${ }^{2}$
}

\begin{abstract}
${ }^{1}$ Laboratory of Cardiovascular Immunology, Key Laboratory of Biological Targeted Therapy of The Ministry of Education, Institute of Cardiology, Union Hospital, Tongji Medical College of Huazhong University of Science and Technology,

Wuhan, Hubei 430000; ${ }^{2}$ Department of Cardiology, The First Affiliated Hospital of Xiamen University,

Xiamen, Fujian 361003; ${ }^{3}$ Key Laboratory of Molecular Biophysics of The Ministry of Education,

Cardio-X Institute, College of Life Science and Technology and Center of Human Genome Research,

Huazhong University of Science and Technology, Wuhan, Hubei 430000, P.R. China
\end{abstract}

Received May 14, 2015; Accepted March 7, 2016

DOI: $10.3892 / \mathrm{mmr} .2016 .5162$

\begin{abstract}
Brugada syndrome (BrS) is an inherited primary arrhythmia disorder, leading to sudden cardiac death due to ventricular tachyarrhythmia, but does not exhibit clinical cardiac abnormalities. The sodium voltage-gated channel $\alpha$ subunit 5 (SCN5A) gene, which encodes the $\alpha$ subunit of the cardiac sodium channel, Nav1.5, is the most common pathogenic gene, although $\geq 22 \mathrm{BrS}$-susceptibility genes have previously been identified. In the present study, a novel genetic variant (p.D1690N) localized in the S5-S6 linker of domain IV of the Nav1.5 channels was identified in a Chinese Han family. Wild-type (WT) and p.D1690N Nav1.5 channels were
\end{abstract}

Correspondence to: Professor Zhengrong Huang or Professor Weihua Li, Department of Cardiology, The First Affiliated Hospital of Xiamen University, 55 Zhenhai Road, Xiamen, Fujian 361003, P.R. China

E-mail: huangzhengrong1@gmail.com

E-mail: weihuaxm@hotmail.com

*Contributed equally

Abbreviations: $\mathrm{I}_{\mathrm{Na}}$, depolarizing inward sodium current; $\mathrm{I}_{\mathrm{Ca}}$, depolarizing inward calcium current; $\mathrm{I}_{\mathrm{Ks}}$, repolarizing outward slow rectifying potassium current; $\mathrm{I}_{\mathrm{to}}$, transient outward potassium current; $I_{\mathrm{Kr}}$, repolarizing outward rapid rectifying potassium current; $I_{\text {KATP }}$, ATP-sensitive inward rectifying potassium current; $I_{f}$, funny current; ChIP, channel-interacting protein; BrS, Brugada syndrome; ECG, electrocardiogram; SCD, sudden cardiac death; PVT, polymorphic ventricular tachycardia; VF, ventricular fibrillation; $\mathrm{RBBB}$, right bundle branch block; ER, endoplasmic reticulum

Key words: Brugada syndrome, sodium voltage-gated channel $\alpha$ subunit 5, sudden cardiac death, genetics, patch-clamp technique, channelopathies transiently over-expressed in HEK293 cells and analyzed via the whole-cell patch clamp technique. The p.D1690N mutation significantly reduced the peak sodium current density to $23 \%$ of WT (at $-20 \mathrm{mV} ; \mathrm{P}<0.01$ ), shifted steady-state activation by $7 \mathrm{mV}$ to increasingly positive potentials $(\mathrm{P}<0.01)$. Furthermore, prolonging of the recovery from inactivation was observed in the p.D1690N mutant. No significant change was identified in steady-state inactivation. Thus, the mutant-induced changes contributed to the loss of function of Nav1.5 channels, which indicates that the p.D1690N variant may have a pathogenic role in $\mathrm{BrS}$.

\section{Introduction}

Brugada syndrome (BrS) is a life-threatening, inherited, primary arrhythmia disorder characterized by ST-segment elevation in the right precordial leads (V1-V3) of the electrocardiogram (ECG), which does not exhibit any clinical cardiac abnormality $(1,2)$. BrS patients may experience syncope and sudden cardiac death (SCD) from episodes of polymorphic ventricular tachycardia (PVT) and ventricular fibrillation (VF), particularly in healthy young males (3). BrS accounts for $4 \%$ of all SCD and $\leq 20 \%$ of sudden deaths in patients without obvious structural heart disease (3). BrS is an inherited disease that shows an autosomal dominant pattern with incomplete penetrance and an incidence ranging from $\sim 5$ per 10,000 in Western countries to 12 per 10,000 in Southeast Asia (3-5).

To date, although 22 susceptibility genes have been identified in BrS, SCN5A, which encodes the $\alpha$ subunit of the major cardiac sodium channel (Nav1.5), is the most common pathogenic gene and is responsible for $11-28 \%$ of $\mathrm{BrS}$ patients (3,6-8). However, mutations in other genes have rarely been observed in $\mathrm{BrS}$ patients and account for the minority $(<25 \%)$ of $\mathrm{BrS}$ genotype-positive cases $(8,9)$. Furthermore, $>370$ mutations in the SCN5A gene have been associated with BrS using a web database, The Gene Connection For The Heart 
(http://triad.fsm.it/cardmoc/). Many of these mutations, which have been characterized in cell lines expressing BrS mutant channels revealed a loss-of-function effect on the sodium current by certain mechanisms, such as reduced current density or disrupted biophysical properties (10-12). Thus, the genetic etiology of BrS remains unclear. Identifying novel susceptibility genes will provide clinical benefit for early diagnosis, risk stratification and personalized treatment of $\mathrm{BrS}$.

The present study investigated a Chinese Han patient presenting with BrS carrying a novel heterozygous mutation, p.D1690N [found in the S5-S6 linker of domain IV (DIV) of the Nav1.5 channel] in the SCN5A gene. In addition, the functional outcomes of the mutated Nav1.5 channel proteins were examined in HEK293 cells. The results demonstrated that the mutation reduced sodium current density and altered the biophysical sodium channel characteristics.

\section{Materials and methods}

Clinical characteristics. A 37-year-old male was admitted to the emergency department of The First Affiliated Hospital of Xiamen University (Xiamen, China) in September 2009, due to sudden syncope from an episode of VF (Fig. 1A). A subsequent 12-lead ECG at rest was consistent with a type-1 Brugada pattern, defined as a prominent coved-type ST-segment elevation, displaying ST-segment elevation $>2 \mathrm{~mm}$ at its peak followed by a negative $\mathrm{T}$ wave (3) and incomplete right bundle branch block (RBBB; Fig. 1B). The BrS patient demonstrated no evidence of structural heart disease by exercise stress test, electrophysiological study and echocardiography. Subsequently, the family of the patient (four

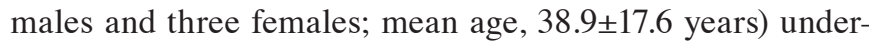
went physical examination, 12-lead ECG, 24-h Holter ECG monitoring (Nihon Kohden, Tokyo, Japan), echocardiography and genetic testing. As ajmaline, flecainide and procainamide, recommended by the consensus report (13), are unobtainable in China, propafenone (Shanghai Xinyi Jinzhu Pharmaceutical Co., Ltd., Shanghai, China) was administered, which has been demonstrated to reveal BrS. Propafenone challenges were performed and evaluated on two of the family members [the sister (II.3) and nephew (III.2) of the proband] as previously described (13). The subjects provided written informed consent and, following a fasting period ( $10 \mathrm{~h}$ overnight), propafenone ( $1 \mathrm{mg} / \mathrm{kg}$ body weight; $10 \mathrm{mg} / \mathrm{min}$ ) was intravenously administered while the patient was continuously monitored by 12-lead ECG and blood pressure. After $20 \mathrm{~min}$, if the reaction was negative (i.e. no change in ECG pattern compared with the baseline, including absence of ST segment elevation and QRS duration prolongation), an additional $0.5 \mathrm{mg} / \mathrm{kg}$ propafenone was injected for $2.5 \mathrm{~min}$. Propafenone infusion was terminated when the diagnostic type I Brugada ECG pattern or ventricular arrhythmias were apparent. After termination of propafenone administration, monitoring was continued for a minimum of $24 \mathrm{~h}$ or until the ECG normalized. The propafenone challenge was performed and evaluated on two family members, II.3 and III.2, who were carriers of the SCN5A mutation.

Genetic analysis. The current study conforms with the principles outlined in the Helsinki Declaration and was approved by the Medical Ethical Committee of The First
Affiliated Hospital of Xiamen University. All subjects provided written informed consent following counseling. Genomic DNA was isolated from leukocyte nuclei using a TIANamp Blood DNA kit (Tiangen Biotech Co., Ltd., Beijing, China), and the cardiac sodium channel gene, SCN5A (transcript, NM_198056.2) was directly sequenced following polymerase chain reaction (PCR) amplification, with the use of an ABI PRISM 3730xl DNA sequencer (Thermo Fisher Scientific, Inc., Waltham, MA, USA) performed by Tsingke Biological Technology Co., Ltd. (Beijing, China), as previously described (12). All DNA-identified variants were compared with a control group of 150 healthy and unrelated Chinese Han individuals (300 alleles) after obtaining written informed consent.

Site-directed mutagenesis and heterologous expression. The p.D1690N mutation was introduced into pcDNA3.1-hH1 using a PCR-based mutagenesis method, as previously described (12). The mutated plasmids were sequenced (Tsingke Biological Technology Co., Ltd.) to ensure the presence of the p.D1690N mutation and the absence of spurious mutations.

The human embryonic kidney 293 cell line, HEK293 was cultured in an incubator in Gibco Dulbecco's modified Eagle's medium (Thermo Fisher Scientific, Inc.) supplemented with Gibco $10 \%$ fetal bovine serum (Thermo Fisher Scientific, Inc.), $4 \mathrm{mmol} / \mathrm{l}$ glutamine (Invitrogen; Thermo Fisher Scientific, Inc.), $100 \mathrm{IU} / \mathrm{ml}$ penicillin (Amresco LLC, Solon, OH, USA) and $100 \mu \mathrm{g} / \mathrm{ml}$ streptomycin (Amresco LLC) at $37^{\circ} \mathrm{C}$ in a humidified atmosphere of $5 \% \mathrm{CO}_{2}$. Prior to over-expression, the cells were seeded in 6-well plates and, upon reaching $80 \%$ confluence, were co-transfected with $0.8 \mu \mathrm{g}$ pcDNA3.1-hH1 or p.D1690N mutant and $0.8 \mu \mathrm{g}$ pIRES2-DsReD-sodium voltage-gated channel $\beta$ subunit 1 (SCNIB; which served as a reporter gene) with Invitrogen Lipofectamine 2000 (Thermo Fisher Scientific, Inc.), according to the manufacturer's instructions and as previously reported (12).

Electrophysiological analysis. Twenty-four to $48 \mathrm{~h}$ after transfection, the sodium current $\left(\mathrm{I}_{\mathrm{Na}}\right)$ was recorded in cells displaying red fluorescence at room temperature $\left(23-25^{\circ} \mathrm{C}\right)$ under whole cell patch-clamp technique, as previously described (12). Briefly, the cells were continuously superfused with a bath solution containing $70 \mathrm{mM} \mathrm{NaCl}, 80 \mathrm{mM} \mathrm{CsCl}$, $5.4 \mathrm{mM} \mathrm{KCl}, 2 \mathrm{mM} \mathrm{CaCl}, 1 \mathrm{mM} \mathrm{MgCl} \mathrm{ma}_{2}, 10 \mathrm{mM}$ HEPES and $10 \mathrm{mM}$ glucose ( $\mathrm{pH}$ adjusted to 7.3 using $\mathrm{CsOH}$ ). The patch pipette (tip resistance, 2-3 M 2 ) was filled with intracellular medium containing $20 \mathrm{mM} \mathrm{NaCl}, 130 \mathrm{mM} \mathrm{CsCl}$, $10 \mathrm{mM}$ HEPES and $10 \mathrm{mM}$ EGTA (pH adjusted to 7.2 with $\mathrm{CsOH}$ ). All the above-mentioned items were purchased from Sigma-Aldrich (St. Louis, MO, USA). The sodium current was recorded using a MultiClamp ${ }^{\mathrm{TM}}$ 700B amplifier (Molecular Devices, LLC, Sunnyvale, CA, USA) and was converted to digital data using a Digidata ${ }^{\circledR} 1440 \mathrm{~A}$ A/D converter $(5 \mathrm{kHz}$ filtering; Molecular Devices, LLC).

Statistical analysis. Continuous results are expressed as means \pm standard error of the mean. Currents were analyzed using Clampfit 10.1 software (Molecular Devices, LLC, Sunnyvale, CA, USA) and Origin Pro 8.5 (OriginLab Corporation,Northampton, MA, USA). Statistical comparisons 
Table I. Susceptibility genes for BrS.

\begin{tabular}{|c|c|c|c|c|}
\hline Type of BrS & Gene & Ion-channel component & Functional effect & Carriers among BrS patients (\%) \\
\hline $\mathrm{BrS} 1$ & SCN5A & $\alpha$ subunit $\mathrm{I}_{\mathrm{Na}}$ & Loss of function & $11-28$ \\
\hline $\mathrm{BrS} 2$ & $G P D 1-L$ & $\mathrm{I}_{\mathrm{Na}} \mathrm{ChIP}$ & Loss of function & $<1$ \\
\hline BrS 3 & CACNAlc & $\alpha$ subunit $\mathrm{I}_{\mathrm{Ca}}$ & Loss of function & $3-4$ \\
\hline $\mathrm{BrS} 4$ & $C A C N B 2 b$ & $\beta$ subunit $\mathrm{I}_{\mathrm{Na}}$ & Loss of function & $2-3$ \\
\hline BrS 5 & $S C N 1 B$ & $\beta$ subunit $\mathrm{I}_{\mathrm{Na}}$ & Loss of function & $<1$ \\
\hline BrS 6 & KCNE3 & $\beta$ subunit $\mathrm{I}_{\mathrm{KS}} / \mathrm{I}_{\mathrm{to}}$ & Gain of function & $<1$ \\
\hline BrS 7 & $S C N 3 B$ & $\beta$ subunit $\mathrm{I}_{\mathrm{Na}}$ & Loss of function & $<1$ \\
\hline $\mathrm{BrS} 8$ & $\mathrm{KCNH} 2$ & $\alpha$ subunit $\mathrm{I}_{\mathrm{Kr}}$ & Gain of function & $<1$ \\
\hline BrS 9 & KCNJ8 & $\alpha$ subunit $\mathrm{I}_{\mathrm{KATP}}$ & Gain of function & $<1$ \\
\hline $\mathrm{BrS} 10$ & CACNA2D1 & $\alpha 2 \delta$ subunit $\mathrm{I}_{\mathrm{Ca}}$ & Loss of function & $<1$ \\
\hline BrS 11 & $R A N G R F$ & $\mathrm{I}_{\mathrm{Na}} \mathrm{ChIP}$ & Loss of function & $<1$ \\
\hline BrS 12 & KCNE5 & $\beta$ subunit $I_{\text {to }}$ & Gain of function & $<1$ \\
\hline $\mathrm{BrS} 13$ & KCND3 & $\alpha$ subunit $I_{\text {to }}$ & Gain of function & $<1$ \\
\hline $\mathrm{BrS} 14$ & HCN4 & $\mathrm{I}_{\mathrm{f}}$ & Loss of function & $<1$ \\
\hline BrS 15 & SLMAP & $\mathrm{I}_{\mathrm{Na}} \mathrm{ChIP}$ & Loss of function & $<1$ \\
\hline BrS 16 & TRMP4 & $\alpha$ subunit & Loss of function & 6 \\
\hline BrS 17 & $S C N 2 B$ & $\beta$ subunit $\mathrm{I}_{\mathrm{Na}}$ & Loss of function & $<1$ \\
\hline BrS 18 & $F G F 12$ & $\mathrm{I}_{\mathrm{Na}} \mathrm{ChIP}$ & Loss of function & $<1$ \\
\hline BrS 19 & $A B C C 9$ & $\mathrm{I}_{\text {KATP }} \mathrm{CHIP}$ & Gain of function & 1 \\
\hline BrS 20 & $P K P 2$ & $\mathrm{I}_{\mathrm{Na}} \mathrm{ChIP}$ & Loss of function & 2.5 \\
\hline $\operatorname{BrS} 21$ & SEMA3A & $\mathrm{I}_{\mathrm{to}} \mathrm{CHIP}$ & Gain of function & 1 \\
\hline $\mathrm{BrS} 22$ & SCN1OA & $\alpha$ subunit $\mathrm{I}_{\mathrm{Na}}$ & Loss of function & 16.7 \\
\hline
\end{tabular}

$\mathrm{I}_{\mathrm{Na}}$, depolarizing inward sodium current; $\mathrm{I}_{\mathrm{Ca}}$, depolarizing inward calcium current; $\mathrm{I}_{\mathrm{Ks}}$, repolarizing outward slow rectifying potassium current; $\mathrm{I}_{\mathrm{to}}$, transient outward potassium current; $\mathrm{I}_{\mathrm{Kr}}$, repolarizing outward rapid rectifying potassium current; $\mathrm{I}_{\mathrm{KATP}}$, ATP-sensitive inward rectifying potassium current; $\mathrm{I}_{\mathrm{f}}$, funny current; ChIP, channel-interacting protein; BrS, Brugada syndrome.

A

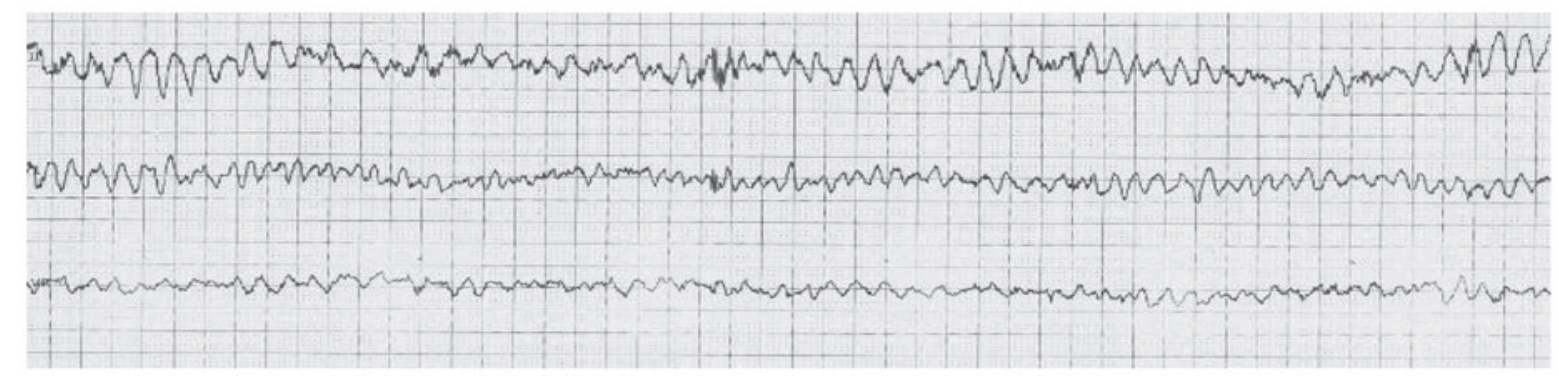

B

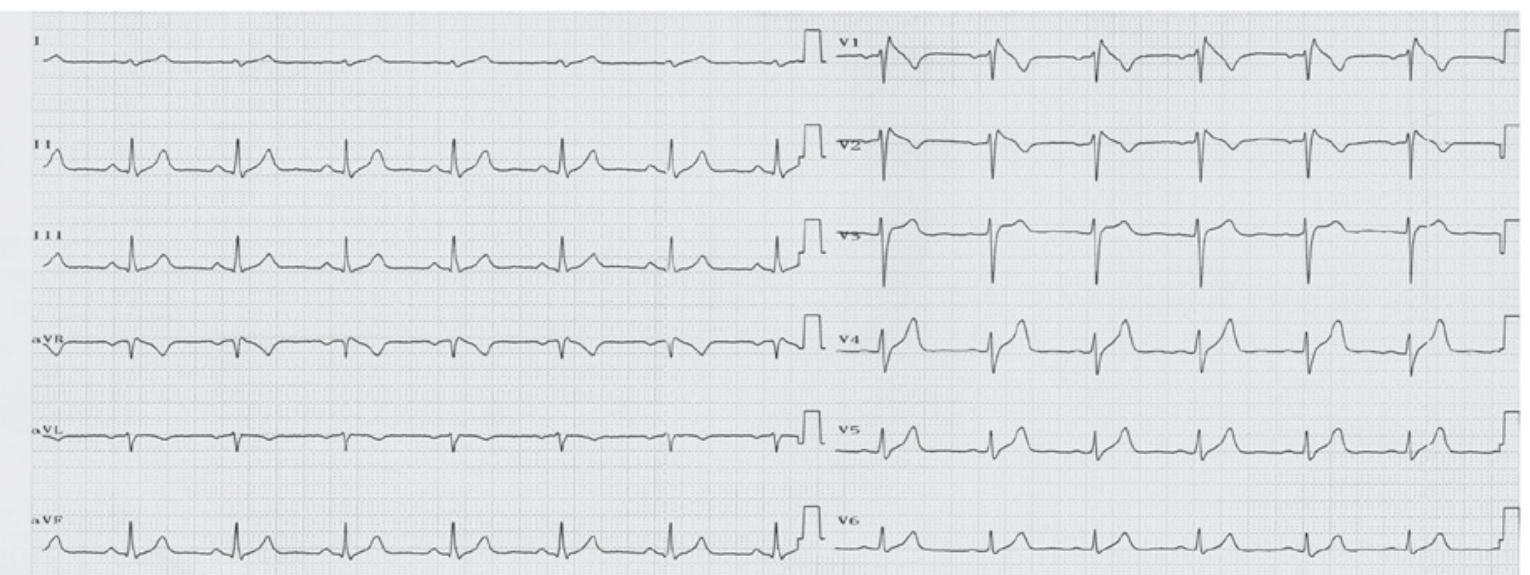

Figure 1. Clinical manifestations of the proband. (A) ECG recording of the proband demonstrating ventricular fibrillation. (B) Representative 12-lead surface ECG recording of the proband showing prominent coved-type ST-segment elevation, following a negative T wave in V1-V2 and incomplete right bundle branch block, which is typically observed in type-1 Brugada syndrome (paper speed, $25 \mathrm{~mm} / \mathrm{sec}$ ). ECG, electrocardiogram. 
between the WT and mutation groups were evaluated using the two-tailed Student's $t$-test and $\mathrm{P}<0.05$ was considered to indicate a statistically significant difference.

\section{Results}

Clinical studies. The present study included seven members of a Chinese Han family with BrS and a history of SCD (the genetic background is presented in Fig. 2A). The proband, a 37-year-old male, was admitted to the emergency department of The First Affiliated Hospital of Xiamen University due to sudden syncopes at night. An ECG demonstrated VF (Fig. 1A), which was treated by electric defibrillation. The clinical history revealed that the patient's father (I.1) succumbed suddenly at the age 53 years. The patient experienced PVT three times in hospital, each of which were successfully treated by electric defibrillation.

At rest, the ECG demonstrated sinus rhythm and was compatible with the type-1 Brugada pattern (Fig. 1B), showing ST-elevation in V1-V3 followed by negative $\mathrm{T}$ wave and RBBB. No structural heart diseases or valvular disease were observed by echocardiography. The proband refused an implantable cardioverter defibrillator, as a result of financial constraints, and succumbed two months later due to SCD. Following complete cardiologic examination, the majority of the patient's relatives exhibited normal ECG patterns and echocardiograms, although the patient's nephew (III.2) exhibited a BrS diagnostic ECG and the patient's sister (II.3) exhibited a negative reaction following propafenone challenge.

Molecular genetic findings. By sequencing SCN5A, a novel missense mutation, c.5262G $>\mathrm{A}$, which was identified in three family members (II.2, II.3 and III.2), is predicted to replace aspartic acid (D) with asparagine $(\mathrm{N})$ at position 1690 (D1690N) in the DIV S5-S6 linker of the Nav1.5 channels (Fig. 2). The variant was not detected in 150 ethnically matched, healthy volunteers (300 alleles) and The Human Gene Mutation Database (14) ruled out the polymorphism.

Additionally, the common single nucleotide polymorphism, c.1673A $>$ G, which produces the p.H558R polymorphism, was not detected in the family members.

Electrophysiology of p.D1690N Nav1.5 channels. To characterize the electrophysiological consequences of the p.D1690N mutation on Nav1.5 channel activity, the biophysical properties of p.D1690N mutant with WT recombinant human sodium channels, combined with the $\mathrm{h} \beta 1$ subunit in HEK293 cell lines, were compared using the whole-cell patch-clamp technique. As shown in Fig. 3A and B, the mutation significantly reduced the peak current densities of Nav1.5 [-20-mV current density: WT $(\mathrm{n}=31),-336.7 \pm 26.9 \mathrm{pA} / \mathrm{pF}$ vs. mutant $(\mathrm{n}=34),-93.3 \pm 9.0 \mathrm{pA} / \mathrm{pF} ; \mathrm{P}<0.01]$. Therefore, it was hypothesized that the position, D1690 may be key in the trafficking and/or stability of the channel.

As shown in Fig. 3C, the steady-state activation and inactivation curves of the WT and mutant were obtained by plotting the normalized peak current as the corresponding membrane voltage. The midpoint of activation $\left(\mathrm{V}_{1} /{ }_{2}\right.$ act $)$ of the p.D1690N mutant was markedly shifted by $7 \mathrm{mV}$ to more positive potentials when compared with the WT channels
$\left[\mathrm{V}_{1} / 2\right.$ act: $-38.2 \pm 1.7 \mathrm{mV},(\mathrm{n}=14)$ vs. $-31.2 \pm 1.6 \mathrm{mV}(\mathrm{n}=12)$ for WT and mutant channels, respectively; $\mathrm{P}<0.01$, Fig. $3 \mathrm{C}]$. By contrast, no significant differences were observed in the voltage dependence of steady-state inactivation $\left[\mathrm{V}_{1} / 2\right.$ inact: $-86.0 \pm 1.5 \mathrm{mV}(\mathrm{n}=15)$ vs. $-82.2 \pm 1.6 \mathrm{mV}(\mathrm{n}=13)$ for $\mathrm{WT}$ and mutant channels, respectively; Fig. $3 \mathrm{C}$ ]. These results revealed that the mutation accelerated activation kinetics. In addition, the recovery constant from fast inactivation for mutant channels was significantly delayed when compared with WT channels $[\tau f=3.9 \pm 0.3 \mathrm{msec}, \tau \mathrm{s}=57.1 \pm 11.0 \mathrm{msec}(\mathrm{n}=14)$ vs. $\tau \mathrm{f}=5.9 \pm 0.4 \mathrm{msec}(\mathrm{P}<0.01$ vs. WT $), \tau \mathrm{s}=73.9 \pm 9.7 \mathrm{msec}(\mathrm{n}=15)$ for WT and mutant channels, respectively; Fig. 3D].

\section{Discussion}

In the present study, the functional consequences of a novel missense mutation, p.D1690N, which was identified in a patient with repeated episodes of $\mathrm{VF}$, and whose ECG presents the typical features of $\mathrm{BrS}$ under basal conditions, was investigated. The electrophysiological analysis revealed that the p.D1690N mutation results in a significant loss of function in the sodium channel, which was predominantly attributable to the reduction in current density and abnormal kinetic properties. Numerous studies indicate that SCN5A mutations associated with BrS result in loss of function of sodium channels $(10,15-17)$. The present results indicate that the $\mathrm{BrS}$ in this particular family was attributed to the novel missense mutation, p.D1690N in the Nav1.5 channels.

$\mathrm{BrS}$ is an inherited channelopathy that is characterized by ST-segment elevation in the right precordial leads (V1-V3) of the electrocardiogram, but that does not demonstrate any cardiac structural disease. To date, $22 \mathrm{BrS}$-susceptibility genes have been identified (Table I), primarily via candidate gene approaches $(3,6-8)$. These genes encode subunits of sodium channels and their interacting proteins [SCN5A, sodium voltage-gated channel $\alpha$ subunit 10 (SCN1OA), SCN1B, sodium voltage-gated channel $\beta$ subunit 2 , sodium voltage-gated channel $\beta$ subunit 3, glycerol-3-phosphate dehydrogenase 1-like, RAN guanine nucleotide release factor, fibroblast growth factor 12, plakophilin 2, sarcolemma associated protein], potassium channels and their interacting proteins (potassium voltage-gated channel subfamily $\mathrm{H}$ member 2, potassium voltage-gated channel subfamily $\mathrm{E}$ regulatory subunit 3, potassium voltage-gated channel subfamily E regulatory subunit 5, potassium voltage-gated channel subfamily D member 3 , potassium voltage-gated channel subfamily $\mathrm{J}$ member 8 , semaphorin 3A, ATP binding cassette subfamily C member 9), L-type calcium channels [calcium voltage-gated channel subunit $\alpha 1 \mathrm{C}$ (CACNA1C), calcium voltage-gated channel auxiliary subunit $\beta$ and calcium voltage-gated channel auxiliary subunit $\alpha 2 \delta 1]$ and other channels (transient receptor potential cation channel subfamily $\mathrm{M}$ member 4 and hyperpolarization activated cyclic nucleotide gated potassium channel 4) $(6,18,19)$. The SCN5A mutations account for $11-28 \%$ of all $\mathrm{BrS}$ patients (8) and the prevalence of the CACNAIC mutation ranges from 2 to $12 \%$ in the literature $(20,21)$. Recently, $\mathrm{Hu}$ et al (9) identified that mutations in the SCN1OA gene, encoding the Nav1.8 channel located adjacent to SCN5A on chromosome 3p21-22, contribute to $16.7 \%$ cases of $\mathrm{BrS}$. However, another study revealed that although SCN1OA variants demonstrated a loss-of-function 
A
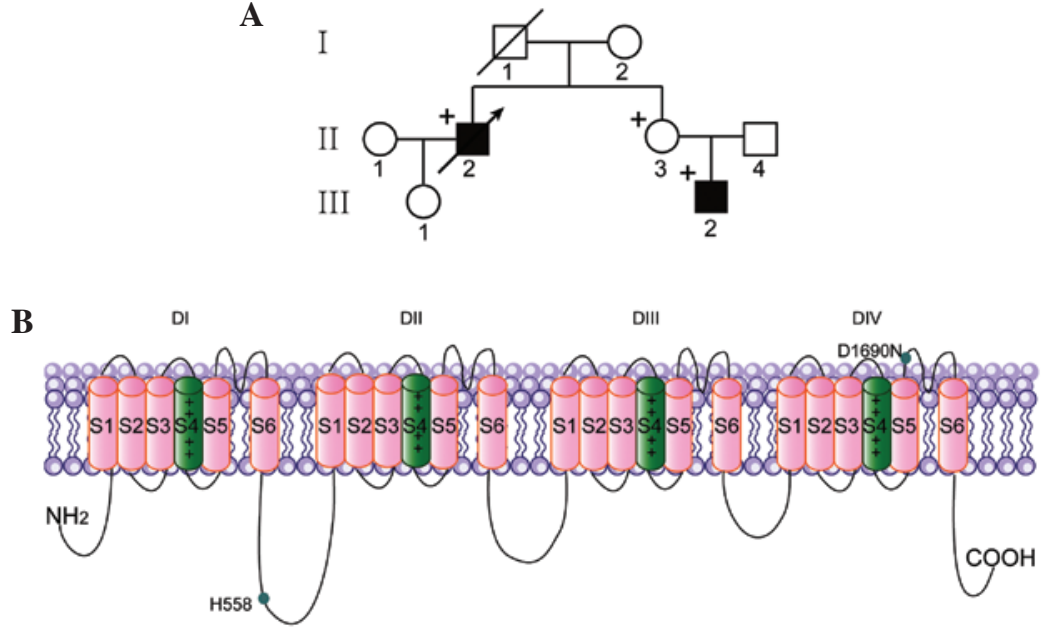

C

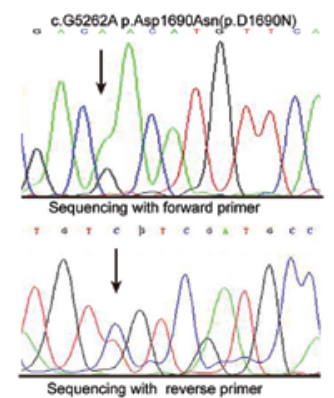

Figure 2. Genetic analysis and background of the proband and family members. (A) The genetic background and mutation status of the Chinese Han family: Males, squares; females, circles; BrS phenotype, filled symbols; variation carrier for SCN5A c.5262G > A, + symbol; proband, arrow. (B) Location of the p.D1690N mutation and the polymorphism in the predicted topological diagram of the Nav1.5 channel. (C) Polymerase chain reaction-based sequence of SCN5A exon 28 demonstrating a heterozygous nucleic acid substitution (c.5262G > A) in the proband, the sister (II.3) and the nephew (III.2), resulting in a single amino acid substitution, p.Asp1690Asn (p.D1690N). SCN5A, sodium voltage-gated channel $\alpha$ subunit 5; D, domain.

A

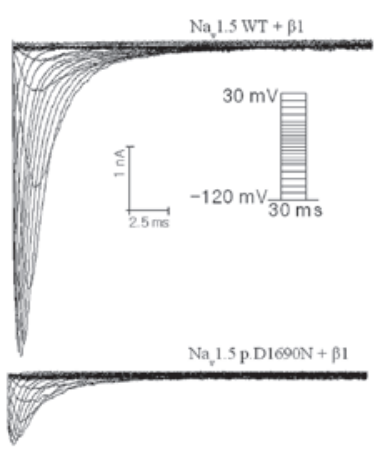

C

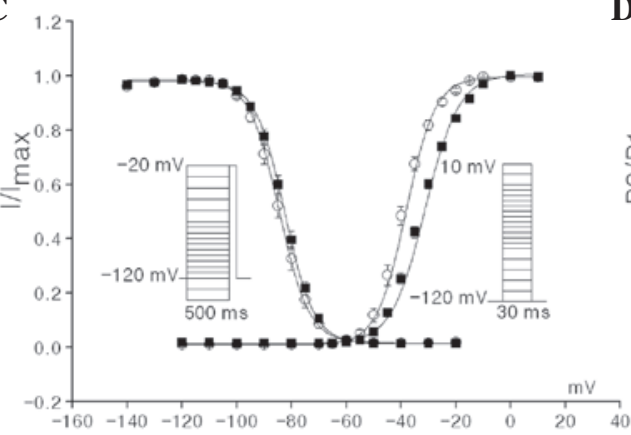

B

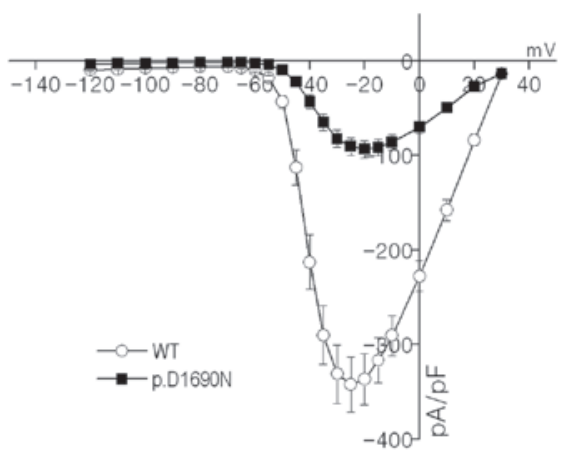

D

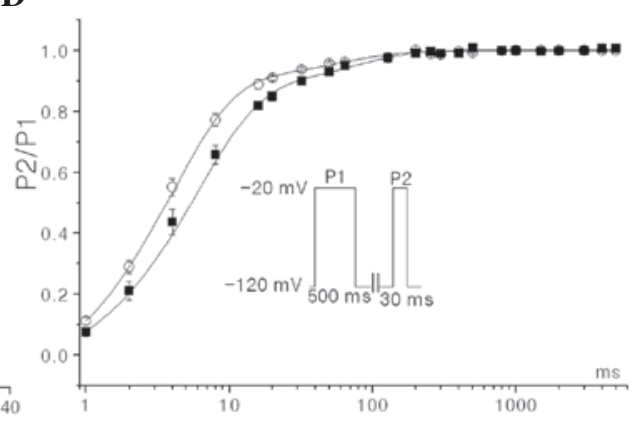

Figure 3. p.D1690N mutation decreases sodium current density and alters the electrophysiological kinetics of Nav1.5 channels. (A) Representative current traces of cells transfected with WT and p.D1690N channels. (B) The current density-voltage association for WT (open circles) and p.D1690N (filled square) channels. (C) Steady-state activation and inactivation curves of WT (open circles) and p.D1690N (filled square) channels obtained by applying the protocol in the inset. (D) Time constants of recovery from fast inactivation was investigated using a twin-pulse protocol (inset); $\tau \mathrm{f}=3.9 \pm 0.3 \mathrm{msec}, \tau \mathrm{s}=57.1 \pm 11.0 \mathrm{msec}$ $(\mathrm{n}=14)$. vs. $\tau \mathrm{f}=5.9 \pm 0.4 \mathrm{msec}(\mathrm{P}<0.01 \mathrm{vs}$. WT $), \tau \mathrm{s}=73.9 \pm 9.7 \mathrm{msec}(\mathrm{n}=15)$ for $\mathrm{WT}$ and mutant channels, respectively. Data are presented as means \pm standard error of the mean. WT, wild-type. 
during in vitro electrophysiological experiments, those variants are not significantly associated with $\mathrm{BrS}$ (22). In addition, novel burden testing revealed that SCN5A alone is responsible for a significant proportion of $\mathrm{BrS}$ cases, and other genes, including SCN1OA and CACNA1C, do not demonstrate enrichment in rare coding variation (6). Thus, this should be noted to avoid diagnosing false-positive cases of causality during genetic counseling, as rare coding variations in BrS-susceptibility genes are also common in healthy individuals.

Genetic screening revealed that two family members of the proband (II.3 and III.2) were carrying the mutation. BrS is a genetically and clinically heterogeneous disease and presents in individuals aged several-months to 80-years-old, although more typically at the average age of 40 years (13). When the manifestations of $\mathrm{BrS}$ are not shown during ECG diagnostics, drug challenge is recommended to reveal asymptomatic first-degree relatives of $\mathrm{BrS}$ patients (13). For the nephew of the proband (III.2; age, 19 years), BrS was definitively diagnosed following a positive propafenone challenge and in conjunction with type 1 pattern ECGs of the family members. The ECG signature of $\mathrm{BrS}$ is dynamic and can be undetected for a period of time. Numerous factors, such as sex hormones, age and fever, have been proposed as being potentially responsible for the phenomenon of $\operatorname{BrS}(23,24)$. Thus, repeated drug challenges or 12-lead, 24-h Holter monitoring are highly recommended for asymptomatic children with a family history of $\operatorname{BrS}(25,26)$.

The p.D1690N mutation causes a negative charge to a neutral amino acid substitution in the pore region between the DIVS5 and DIVS6 transmembrane segments that significantly affects channel expression and gating kinetics. A central ion-conducting pore of Nav1.5 channels is formed by the S5 and S6 segments and their linker extracellular loops, determining ion selectivity. Núñez et al (15) identified two p.D1690N and p.G1748D compound heterozygous mutations in the Nav1.5 channel in a Caucasian family with a history of BrS. Notably, p.D1690N completely restores the gating defects presented by p.G1748D channels; however, has little impact on its trafficking. However, the p.D1690N mutation alone has not been identified in BrS patients. In the present study, a missense variant, p.D1690N was identified in the Nav1.5 channel in three of the proband's family members (II.2, II.3 and III.2). During comparisons of the electrophysiological characteristics of the WT and p.D1690N mutant in HEK293 cells, it was identified that the key alteration of the p.D1690N mutation was the dramatic reduction in $\mathrm{I}_{\mathrm{Na}}$ density $(\sim 72 \%)$ predominantly attributable to a significant impairment of channel trafficking toward the membrane. In addition, the p.D1690N mutant delayed the time of channel recovery from inactivation induced by depolarization. These changes caused by the p.D1690N mutation may reduce the activity of the Nav1.5 channel and contribute to BrS.

There have been various studies investigating the underlying mechanisms of decreasing current density by missense mutations. One mechanism is that the mutant channels fail to properly traffic to the cytomembrane and are detained in the endoplasmic reticulum (ER) by a quality control system, such as R1432G (11). The quality control system in the ER initially arrested and ultimately degraded the mutation-induced incorrectly folded proteins (11). A second mechanism is that certain mutations, localized in the ion-conducting pore, may block
$\mathrm{Na}^{+}$permeation, inducing a significant reduction in the sodium current through the channels (27). Consistent with previous results, the present study identified that the mechanism underlying the reduction in sodium current density is the trafficking disorders due to the p.D1690N mutation.

A previous report demonstrated that pharmacological interventions (such as administration of mexiletine) significantly increased current density in a G1743R mutant by rescuing its expression levels in the plasma membrane (28). Although pharmacological interventions provide potential clinical benefit to BrS patients carrying the mutation, whether the p.D1690N mutant channel may be rescued by mexiletine administration requires further investigation. In addition, various studies revealed that the $\mathrm{p} . \mathrm{H} 558 \mathrm{R}$ polymorphism restores the biophysical defects caused by numerous loss-of-function SCN5A mutations underlying sudden infant death syndrome, cardiac conduction disease and BrS (29-31). In the SCN5A mutations associated with BrS, p.H558R increases the current density generated by p.R282H (32), p.D1690N (15), p.S216L (33) and p.K317N (34), predominantly by mitigating their impaired trafficking. Thus, the absence of p.H558R in this family fails to restore the trafficking defect in D1690N in the SCN5A gene encoding cardiac $\mathrm{Na}^{+}$channels (such as Nav1.5).

In conclusion, a novel heterozygous human mutation (D1690N), in the DIV S5-S6 linker of the Nav1.5 channel, was identified in a Chinese Han patient with BrS and the patient's family. The results indicate that the marked reduction of sodium current density in the p.D1690N mutant is attributable to trafficking disorders of the Nav1.5 channel proteins to the sarcolemma. The decreased sodium current density and abnormal activities caused by the p.D1690N mutation are consistent with the hypothesis that a loss of function of Nav1.5 contributes to the pathogenesis of BrS. Whether the characterizations of the p.D1690N mutant exist in vivo and the precise mechanisms require further research. However, the present study strengthens the understanding of the association between the structure and function of the Nav1.5 channel, and provides potential personalized therapeutic approaches for inherited cardiac arrhythmia.

\section{Acknowledgements}

The authors would like to thank Dr Qing K. Wang (Department of Molecular Cardiology, Lerner Research Institute, Cleveland Clinic, Cleveland, OH, USA) for assistance with the present study and for providing the pcDNA3.1-hH1 and pIRES2-DsReD-SCN1B plasmids. The present study was supported by the National Natural Science Foundation of China (grant nos. 81270277 and 81170090).

\section{References}

1. Chen PS and Priori SG: The Brugada Syndrome. J Am Coll Cardiol 51: 1176-1180, 2008.

2. Brugada P and Brugada J: Right bundle branch block, persistent ST segment elevation and sudden cardiac death: A distinct clinical and electrocardiographic Syndrome. A multicenter report. J Am Coll Cardiol 20: 1391-1396, 1992.

3. Antzelevitch C, Brugada P, Borggrefe M, Brugada J, Brugada R, Corrado D, Gussak I, LeMarec H, Nademanee K, Perez Riera AR, et al: Brugada syndrome: Report of the second consensus conference: Endorsed by the heart rhythm society and the European heart rhythm association. Circulation 111: 659-670, 2005. 
4. Probst V, Veltmann C, Eckardt L, Meregalli PG, Gaita F, Tan HL, Babuty D, Sacher F, Giustetto C, Schulze-Bahr E, et al: Long-term prognosis of patients diagnosed with brugada syndrome: Results from the FINGER brugada syndrome registry. Circulation 121: 635-643, 2010.

5. Miyasaka Y, Tsuji H, Yamada K, Tokunaga S, Saito D, Imuro Y, Matsumoto $\mathrm{N}$ and Iwasaka T: Prevalence and mortality of the Brugada-type electrocardiogram in one city in Japan. J Am Coll Cardiol 38: 771-774, 2001.

6. Le Scouarnec S, Karakachoff M, Gourraud JB, Lindenbaum P, Bonnaud S, Portero V, Duboscq-Bidot L, Daumy X, Simonet F, Teusan $\mathrm{R}$, et al: Testing the burden of rare variation in arrhythmia-susceptibility genes provides new insights into molecular diagnosis for Brugada Syndrome. Hum Mol Genet 24: 2757-2763, 2015

7. Wang Q, Ohno S, Ding WG, Fukuyama M, Miyamoto A, Itoh H, Makiyama T, Wu J, Bai J, Hasegawa K, et al: Gain-of-function KCNH2 mutations in patients with Brugada Syndrome. J Cardiovasc Electrophysiol 25: 522-530, 2014.

8. Kapplinger JD, Tester DJ, Alders M, Benito B, Berthet M, Brugada J, Brugada P, Fressart V, Guerchic off A, Harris-Kerr C, et al: An international compendium of mutations in the SCN5A-encoded cardiac sodium channel in patients referred for Brugada Syndrome genetic testing. Heart Rhythm 7: 33-46, 2010.

9. Hu D, Barajas-Martínez H, Pfeiffer R, Dezi F, Pfeiffer J, Buch T, Betzenhauser MJ, Belardinelli L, Kahlig KM, Rajamani S, et al: Mutations in SCN10A are responsible for a large fraction of cases of Brugada Syndrome. J Am Coll Cardiol 64: 66-79, 2014.

10. Zimmer T and Surber R: SCN5A channelopathies-an update on mutations and mechanisms. Prog Biophys Mol Biol 98: 120-136, 2008

11. Baroudi G, Pouliot V, Denjoy I, Guicheney P, Shrier A and Chahine M: Novel mechanism for Brugada Syndrome: Defective surface localization of an SCN5A mutant (R1432G). Circ Res 88: E78-E83, 2001

12. Zeng Z, Zhou J, Hou Y, Liang X, Zhang Z, Xu X, Xie Q, Li W and Huang Z: Electrophysiological characteristics of a SCN5A voltage sensors mutation R1629Q associated with Brugada syndrome. PLoS One 8: e78382, 2013.

13. Wilde AA, Antzelevitch C, Borggrefe M, Brugada J, Brugada R, Brugada P, Corrado D, Hauer RN, Kass RS, Nademanee K, et al: Proposed diagnostic criteria for the Brugada Syndrome: Consensus report. Circulation 106: 2514-2519, 2002.

14. Stenson PD, Mort M, Ball EV, Shaw K, Phillips A and Cooper DN: The human gene mutation database: Building a comprehensive mutation repository for clinical and molecular genetics, diagnostic testing and personalized genomic medicine. Hum Genet 133: 1-9, 2014.

15. Núñez L, Barana A, Amorós I, de la Fuente MG, Dolz-Gaitón P, Gómez R, Rodríguez-García I, Mosquera I, Monserrat L, Delpón E, et al: p.D1690N Nav1.5 rescues p.G1748D mutation gating defects in a compound heterozygous Brugada Syndrome patient. Heart Rhythm 10: 264-272, 2013.

16. Lin YJ, Higa S, Tai CT, Chang SL, Lee KT, Lo LW, Ishigaki S, Tuan TC, Wongcharoen W, Hu YF, et al: Role of the right atrial substrate in different types of atrial arrhythmias. Heart Rhythm 6: 592-598, 2009.

17. Chiang KC, Lai LP and Shieh RC: Characterization of a novel Nav1.5 channel mutation, A551T, associated with Brugada Syndrome. J Biomed Sci 16: 76, 2009.

18. Boczek NJ, Ye D, Johnson EK, Wang W, Crotti L, Tester DJ, Dagradi F, Mizusawa Y, Torchio M, Alders M, et al: Characterization of SEMA3A-encoded semaphorin as a naturally occurring Kv4.3 protein inhibitor and its contribution to Brugada Syndrome. Circ Res 115: 460-469, 2014.
19. Saber S, Amarouch MY, Fazelifar AF, Haghjoo M, Emkanjoo Z, Alizadeh A, Houshmand M, Gavrilenko AV, Abriel $\mathrm{H}$ and Zaklyazminskaya EV: Complex genetic background in a large family with Brugada Syndrome. Physiol Rep 3: e12256, 2015.

20. Burashnikov E, Pfeiffer R, Barajas-Martinez H, Delpón E, $\mathrm{Hu}$ D, Desai M, Borggrefe M, Häissaguerre M, Kanter R, Pollevick GD, et al: Mutations in the cardiac L-type calcium channel associated with inherited J-wave Syndromes and sudden cardiac death. Heart Rhythm 7: 1872-1882, 2010.

21. Wilde AA and Behr ER: Genetic testing for inherited cardiac disease. Nat Rev Cardiol 10: 571-583, 2013.

22. Behr ER, Savio-Galimberti E, Barc J, Holst AG, Petropoulou E, Prins BP, Jabbari J, Torchio M, Berthet M, Mizusawa Y, et al; UK10K Consortium and Jamshidi Y: Role of common and rare variants in SCN10A: Results from the Brugada syndrome QRS locus gene discovery collaborative study. Cardiovasc Res 106: $520-529,2015$

23. Shimizu W, Matsuo K, Kokubo Y, Satomi K, Kurita T, Noda T, Nagaya N, Suyama K, Aihara N, Kamakura S, et al: Sex hormone and gender difference-role of testosterone on male predominance in Brugada Syndrome. J Cardiovasc Electrophysiol 18: 415-421, 2007.

24. Junttila MJ, Gonzalez M, Lizotte E, Benito B, Vernooy K, Sarkozy A, Huikuri HV, Brugada P, Brugada J and Brugada R: Induced Brugada-type electrocardiogram, a sign for imminent malignant arrhythmias. Circulation 117: 1890-1893, 2008.

25. Conte G, de Asmundis C, Ciconte G, Julià J, Sieira J, Chierchia GB and Brugada P: Follow-up from childhood to adulthood of individuals with family history of Brugada syndrome and normal electrocardiograms. JAMA 312: 2039-2041, 2014.

26. Cerrato N, Giustetto C, Gribaudo E, Richiardi E, Barbonaglia L, Scrocco C,Zema D and Gaita F: Prevalence of type 1 brugada electrocardiographic pattern evaluated by twelve-lead twenty-four-hour holter monitoring. Am J Cardiol 115: 52-56, 2015.

27. Amin AS, Verkerk AO, Bhuiyan ZA, Wilde AA and Tan HL: Novel Brugada syndrome-causing mutation in ion-conducting pore of cardiac Na+ channel does not affect ion selectivity properties. Acta Physiol Scand 185: 291-301, 2005.

28. Valdivia CR, Tester DJ, Rok BA, Porter CB, Munger TM, Jahangir A, Makielski JC and Ackerman MJ: A trafficking defective, Brugada syndrome-causing SCN5A mutation rescued by drugs. Cardiovase Res 62: 53-62, 2004.

29. Lizotte E, Junttila MJ, Dube MP, Hong K, Benito B, DE Zutter M, Henkens S, Sarkozy A, Huikuri HV, Towbin J, et al: Genetic modulation of brugada Syndrome by a common polymorphism. J Cardiovasc Electrophysiol 20: 1137-1141, 2009.

30. Viswanathan PC, Benson DW and Balser JR: A common SCN5A polymorphism modulates the biophysical effects of an SCN5A mutation. J Clin Invest 111: 341-346, 2003.

31. Shinlapawittayatorn K, Du XX, Liu H, Ficker E, Kaufman ES and Deschênes I: A common SCN5A polymorphism modulates the biophysical defects of SCN5A mutations. Heart Rhythm 8: 455-462, 2011.

32. Poelzing S, Forleo C, Samodell M, Dudash L, Sorrentino S, Anaclerio M, Troccoli R, Iacoviello M, Romito R, Guida P, et al: SCN5A polymorphism restores trafficking of a Brugada syndrome mutation on a separate gene. Circulation 114: 368-376, 2006.

33. Marangoni S, Di Resta C, Rocchetti M, Barile L, Rizzetto R, Summa A, Severi S, Sommariva E, Pappone C, Ferrari M, et al: A Brugada syndrome mutation (p.S216L) and its modulation by p.H558R polymorphism: Standard and dynamic characterization. Cardiovasc Res 91: 606-616, 2011.

34. Shinlapawittayatorn K, Dudash LA, Du XX, Heller L, Poelzing S, Ficker E and Deschênes I: A novel strategy using cardiac sodium channel polymorphic fragments to rescue trafficking-deficient SCN5A mutations. Circ Cardiovasc Genet 4: 500-509, 2011. 